\title{
JMSR
}

Journal of Medical and Scientific Research

\section{Does elevated red cell distribution width shows association with increased Framingham risk score among patients with coronary artery disease? - A cross-sectional study}

Meenakshi R. Shah ${ }^{1}$, Varsha Y. Godbole ${ }^{1}$, and Kedar G. Mehta ${ }^{2, *}$

${ }^{1}$ Department of Medicine, GMERS Medical College, Gotri, Vadodara, Gujarat -390021, India

${ }^{2}$ Department of Community Medicine, GMERS Medical College, Gotri, Vadodara, Gujarat -390021, India

\begin{abstract}
Background: Red-cell distribution width (RDW) is one of the routinely done investigations in complete blood count. Various studies have found that increased RDW has been associated with adverse outcomes in cardiac diseases. Framingham risk score (FRS) is also one of the widely used risk score for assessment in coronary artery disease (CAD) patients. This study was conducted with an objective to find association between RDW and FRS in patients with coronary artery disease. This cross-sectional study was conducted among patients visiting tertiary care hospital in Vadodara, Gujarat.
\end{abstract}

Material and methods: A total of 204 patients (104 CAD cases and 100 controls) were enrolled in the study. Clinical and demographic characteristics were obtained from all patients. FRS was calculated on patients in both the groups. Investigations like CBC testing, renal function and lipid profile were also conducted for patients in both the groups. Descriptive statistics for baseline characteristics, clinical and haematological parameters have been mentioned in the form of mean and standard deviation. Association between clinical, haematological parameters and FRS with RDW among CAD patients is calculated by correlation coefficient $(r)$.

Results: Baseline characteristics of the patients like gender, BMI and diabetes were similar in both the CAD and the control group. RDW was significantly correlated with the FRS ( $p<0.0001), R B C$ count $(p<0.001), M C V$ $(p<0.0001)$ and MCH $(p<0.008)$ in CAD patients. However, RDW was not statistically different in the patients of CAD having other co-morbidities like diabetes and/or hypertension.

Conclusion: There is significant association between the elevated RDW and increased FRS which suggested an increased risk of cardiac events in patients with coronary artery disease. As compared to other biomarkers it is a simple, rapid and inexpensive marker so it can be routinely prescribed among CAD patients.

Keywords: red cell distribution; Framingham risk score; coronary artery disease; complete blood count.

*Corresponding author: Dr. Kedar Mehta, Department of Community Medicine, GMERS Medical College, Gotri, Vadodara, Gujarat -390021, India. Mobile number: 9879036835; Email: kedar_mehta20@yahoo.co.in

Received 20 July 2020; Revised 29 August 2020; Accepted 5 September 2020; Published 12 September 2020

Citation: Shah MR, Godbole VY, Mehta KG. Does elevated red cell distribution width shows association with increased Framingham risk score among patients with coronary artery disease? - A crosssectional study. J Med Sci Res. 2020; 8(4):140-144. DOI: http:// dx.doi.org/10.17727/JMSR.2020/8-18

Copyright: (C) 2020 Shah MR et al. Published by KIMS Foundation and Research Center. This is an open-access article distributed under the terms of the Creative Commons Attribution License, which permits unrestricted use, distribution, and reproduction in any medium, provided the original author and source are credited. 


\section{Introduction}

Red-cell distribution width (RDW) indicates variability in the size of circulatory erythrocytes. It is routinely done as a part of complete blood count [1]. Many studies have found association of increased RDW with adverse outcomes in cardiac, renal and infectious diseases [2-6].

Amongst various non-communicable diseases, coronary artery disease (CAD) is one of the leading causes of morbidity and mortality. Coronary angiography continues to remain gold standard for diagnosis of CAD. However, various noninvasive risk models are developed to predict the cardiovascular risk among different patients. One such predictor risk model is Framingham risk score (FRS) which has been developed from the findings of Framingham heart study. FRS current version of 2008 has been validated in various studies and we are not aware of any other importance of FRS apart from risk assessment for CAD among patients. RDW measurement is relatively simple and cost-effective and one of the routinely used investigations as a part of complete blood count. So, the present study was conducted with objectives to determine association of RDW with FRS among patients with coronary artery disease.

\section{Materials and methods}

Ethics approval: The study was approved by the institutional ethics committee of GMERS Medical College and Hospital Vadodara, Gujarat. All the patients were enrolled in the study after taking written informed consent.

\section{Method}

A total of 204 patients were recruited in the study. All the patients had undergone ECG, Echo and/or coronary angiography for confirmation of diagnosis of CAD and then were labelled as 'Cases'. Their clinical and haematological parameters were noted in their individual case record forms.

Patients with acute infections, inflammatory disease, renal disease, neoplasm, recent myocardial infarction or congestive heart failure were excluded from the study.

The patients were considered to be having CAD if they had a history of acute myocardial infarction at least three months before, or had angiographically proven CAD or there was evidence of regional wall motion abnormality on echocardiography. These patients formed the CAD case group ( $\mathrm{n}=104)$. Those without evidence of CAD formed the control group $(n=100)$. Clinical and demographic details of all patients were noted in their individual case record forms. The FRS was calculated on patients in both cases and control groups. CBC testing, renal function tests and lipid profile were also done with appropriate automated analysers for patients of both groups.

\section{Statistical analysis}

Data was entered in Microsoft excel worksheet and double data entry was done to maintain accuracy and completeness of data. All the data files were kept password protected to ensure confidentiality. Data was then analysed using EpiInfo software version 7.2.2.6.

Descriptive statistics for baseline characteristics, clinical and haematological parameters have been mentioned in the form of mean and standard deviation. Quantitative variables between CAD and normal subjects were tested using unpaired $t$ test and $p$ value of less than 0.05 is statistically significant. We have also tried to find association between clinical and haematological parameters with RDW among CAD patients by correlation coefficient $(r)$ and $p$ value of $r$, less than 0.05 is considered as statistically significant association.

\section{Results}

Clinical and demographic characteristics of the patients with CAD and the control group are shown in Table 1. A total of 204 patients were enrolled in the study including 104 cases viz. CAD patients and 100 controls. The mean age of patients was $60 \pm 9$ years in the $\mathrm{CAD}$ group and $51 \pm 8.5$ years in the control group. With regards to variables like gender, smoking, alcohol and BMI there was no statistically significant difference between both the groups. Hypertension was significantly higher in the CAD group than the control group $(p<0.0001)$. Comparison of clinical and haematological parameters between cases and control are shown in Table 2. There was no statistical difference between the groups for haematological parameters like hematocrit, MCV, platelet count, random blood sugar (RBS) and serum creatinine levels. On comparing lipid profile, total cholesterol 
and LDL cholesterol levels were higher in CAD group as compared to the control group. The mean FRS was higher in the CAD group $(29.9 \pm 20.5)$ as compared to control group (13.5 \pm 10.9 ), which was statistically significant $(\mathrm{p}<0.0001)$. The mean RDW was $16.8 \pm$ 2.1 in the CAD group and $14.4 \pm 2.3$ in the control group which was also statistically significant.

Table 1: Clinical and Demographic characteristics of study participants (Cases - CAD patients and control subjects).

\begin{tabular}{|lcc|}
\hline \multicolumn{1}{|c}{ Variables } & $\begin{array}{c}\text { CAD Patients } \\
(\mathrm{N}=104)\end{array}$ & $\begin{array}{c}\text { Control group } \\
(\mathrm{N}=100)\end{array}$ \\
\hline Age & $60.16 \pm 9.14$ & $51.14 \pm 8.56$ \\
Male & 67 & 62 \\
Female & 37 & 32 \\
BMI & $24.19 \pm 2.99$ & $26.79 \pm 4.78$ \\
Smoking & 38 & 8 \\
Alcohol & 17 & 18 \\
Hypertension & 73 & 38 \\
Diabetes & 39 & 42 \\
\hline
\end{tabular}

Correlation was done between RDW and various clinical and haematological parameters including FRS in CAD patients as shown in Table 3. RDW was significantly correlated with the RBC count $(\mathrm{p}<0.001), \operatorname{MCV}(\mathrm{p}<0.0001)$ and MCH $(\mathrm{p}<0.008)$ in CAD patients. There was a significant correlation between RDW and FRS ( $\mathrm{p}<0.0001)$ among CAD patients. Other parameters like BMI, Haemoglobin levels, WBC, platelet count, random blood glucose level, Serum creatinine levels and LDL cholesterol did not show statistical significance with RDW. The RDW was not statistically different in the patients of CAD having other co-morbidities like diabetes and/ or hypertension (Table 4).

\section{Discussion}

RDW is an important indicator of RBC size variation. Our study showed statistically significant association of increased RDW with high FRS score among CAD patients. Moreover, in our study both FRS and RDW were significantly higher in the CAD group when compared to the control group.

Table 2: Comparison of clinical and hematological profile among cases (CAD patients) and control subjects.

\begin{tabular}{|c|c|c|c|c|c|}
\hline \multirow{2}{*}{ Variables } & \multicolumn{2}{|c|}{ CAD Patients $(\mathrm{N}=104)$} & \multicolumn{2}{|c|}{ Control group $(N=100)$} & \multirow{2}{*}{ P value } \\
\hline & Mean & $S D$ & Mean & $S D$ & \\
\hline Framingham risk score & 29.98 & 20.57 & 13.52 & 10.96 & $<0.0001^{*}$ \\
\hline Body mass index $\left(\mathrm{kg} / \mathrm{m}^{2}\right)$ & 24.19 & 2.99 & 24.79 & 2.89 & 0.08 \\
\hline Hemoglobin (gm\%) & 13.58 & 1.01 & 13.07 & 0.96 & $<0.001^{*}$ \\
\hline Red blood cell count & 4.88 & 0.44 & 4.68 & 0.66 & $0.02^{*}$ \\
\hline White blood cell count & 8080.01 & 2573.60 & 6400.70 & 1792.31 & $<0.0001^{*}$ \\
\hline Neutrophil & 68.16 & 7.81 & 66.07 & 6.75 & $0.04^{*}$ \\
\hline Lymphocytes & 24.59 & 7.05 & 26.11 & 5.82 & 0.08 \\
\hline Platelets & 2.72 & 0.80 & 2.74 & 0.79 & 0.91 \\
\hline MCV & 82.72 & 5.62 & 81.28 & 7.70 & 0.09 \\
\hline $\mathrm{MCH}$ & 29.92 & 2.77 & 27.46 & 2.96 & $<0.0001^{*}$ \\
\hline RDW & 16.83 & 2.10 & 14.49 & 2.38 & $<0.001^{*}$ \\
\hline Random blood sugar & 130.42 & 51.38 & 133.63 & 57.37 & 0.29 \\
\hline S. creatinine & 1.07 & 0.29 & 1.01 & 0.32 & 0.08 \\
\hline Total cholesterol & 184.24 & 52.27 & 167.82 & 64.43 & $0.04^{*}$ \\
\hline LDL cholesterol & 114.23 & 34.58 & 100.26 & 55.41 & $0.02^{*}$ \\
\hline HDL cholesterol & 36.79 & 8.38 & 36.80 & 12.28 & 0.98 \\
\hline Triglycerides & 135.04 & 39.24 & 128.54 & 35.14 & 0.21 \\
\hline
\end{tabular}

*statistically significant 
Table 3: Correlation of hematological and clinical variables with RDW among patients with CAD patients $(\mathrm{N}=104)$.

\begin{tabular}{|lcc|}
\hline \multirow{2}{*}{ Variables } & \multicolumn{2}{c|}{ RDW } \\
\cline { 2 - 3 } & $\begin{array}{c}r \text { (correlation } \\
\text { coefficient) }\end{array}$ & P value \\
\hline Age & 0.092 & 0.36 \\
Framingham risk score & 0.516 & $<0.0001^{*}$ \\
Body mass index (kg/m ${ }^{2}$ ) & 0.183 & 0.06 \\
Hemoglobin (gm\%) & 0.092 & 0.36 \\
Red blood cell count & 0.437 & $<0.0001^{*}$ \\
White blood cell count & 0.106 & 0.295 \\
Neutrophil & 0.065 & 0.519 \\
Lymphocytes & -0.172 & 0.08 \\
Platelets & 0.102 & 0.314 \\
MCV & -0.516 & $<0.0001^{*}$ \\
MCH & -0.329 & $0.0008^{*}$ \\
Random blood sugar & 0.051 & 0.611 \\
S. creatinine & 0.135 & 0.18 \\
Total cholesterol & -0.210 & $0.03^{*}$ \\
LDL cholesterol & -0.115 & 0.25 \\
HDL cholesterol & 0.166 & 0.09 \\
Triglycerides & 0.152 & 0.131 \\
\hline
\end{tabular}

*statistically significant

Table 4: Association of RDW with other comorbidities among study participants.

\begin{tabular}{|lcc|}
\hline & RDW & Pvalue \\
\hline DM & $15.5 \pm 2.10$ & \\
HT & $14.9 \pm 1.98$ & 0.09 \\
DM and HT & $15.8 \pm 2.15$ & \\
\hline
\end{tabular}

It is already known that an elevated FRS is associated with increased risk of patients to develop coronary artery disease and it is a well-established and validated risk assessment tool. In our study RDW is significantly associated with FRS and so we can simply look at RDW values to evaluate the risk of coronary artery disease. Increased RDW is observed among anaemic patients and other haematological problems so such patients were excluded from our study. There was no significant difference in the RDW amongst patients with co-morbidities like diabetes and hypertension as compared to those not having them. Various studies have concluded that RDW is independently associated with cardiac failure and hence associated with cardiac morbidity and mortality [7-9]. A study done by Ani et al. found association of increased RDW among stroke patients with increased morbidity [10]. Similar finding was noted that elevated RDW was associated with severity of peripheral vascular diseases $[11,12]$.

Many previous studies have proposed the concept of elevated RDW as a prognostic marker for patients with CAD [13-16]. Studies in the general population have shown an association between atherosclerosis and an elevated RDW. Increased RDW is associated with metabolic syndrome, a known risk factor in cardiovascular disease $[17,18]$.

The exact mechanisms underlying this association though remain elusive. The prognostic value of RDW results due to it being an inflammatory marker. Chronic inflammation is the basis of atherosclerosis and its complications including upregulation of CRP, TNF receptor and IL levels. Additionally, changes in osmolality under some conditions decreases the ability of RBCs to deform leading to increased RDW level. This results in decreased microvascular perfusion and increased CVDs. In CVDs, the inflammation and oxidative stress can increase RDW level by iron metabolism and reducing RBC lifespan. Some studies have found increased RDW with inflammatory markers such as CRP, TNF receptor and IL-S levels [19]. Thus, the increased RDW may be a reflection of underlying chronic inflammatory state which may predispose to increased risk of CAD.

Our study shows statistically significant association of high hemoglobin, WBC count and MCH with CAD. Many studies have shown that RBC count, WBC count and platelets, play a significant role in the development and prediction of recurrence and death in CAD $[20,21]$. High Hemoglobin values and WBC count are indicators of inflammation and are important in the pathogenesis of CAD. In our study, $\mathrm{MCH}$ as a variable is correlating with CAD patients (Table 2). Various studies have shown that increased WBC count, RBC count, neutrophil \%, MCV and MCHC are associated with poor prognosis in CAD patients [20, 21]. MCH may also correlate with the severity, however we did not find any studies regarding the same. 


\section{Conclusion}

We found an association between the elevated RDW and increased FRS which suggested an increased risk of cardiac events in patients with coronary artery disease. As compared to other biomarkers it is a simple, rapid and inexpensive markers it is automatically generated during routine study. The drawbacks of the study are the standardisation of the RDW values which are generated in different haematology analysers. Another question with RDW is to know whether the association is casual or just a mirror reflection of the underlying metabolic derangement. Irrespectively in our resource stressed country where cost of investigations can be a huge burden to many people it is worthwhile to broaden the role of RDW beyond haematology and look for its importance in diagnosis and prognosis of patients with coronary artery disease.

\section{Conflicts of interest}

Authors declare no conflicts of interest.

\section{References}

[1] Salvagno GL, Sanchis-Gomar F, Picanza A, Lippi G. Red blood cell distribution width: A simple parameter with multiple clinical applications. Crit Rev Clin Lab Sci. 2015; 52(2):86105.

[2] Sičaja M, Pehar M, DerekL, Starčević B, Vuletić V, et al. Red blood cell distribution width as a prognostic marker of mortality in patients on chronic dialysis: a single center, prospective longitudinal study. Croat Med J. 2013; 54(1):2532.

[3] Li W, Li X, Wang M, Ge X, Li F, et al. Association between red cell distribution width and the risk of heart events in patients with coronary artery disease. Exp Ther Med. 2015; 9(4):1508-1514.

[4] Chen B, Ye B, Zhang J, Ying L, Chen Y. RDW to platelet ratio: a novel noninvasive index for predicting hepatic fibrosis and cirrhosis in chronic hepatitis B. PLoS One. 2013; 8:e68780.

[5] Lee JH, Chung HJ, Kim K, Jo YH, Rhee JE, et al. Red cell distribution width as a prognostic marker in patients with community-acquired pneumonia. Am J Emerg Med. 2013; 31(1):72-79.

[6] Şenol K, Saylam B, Kocaay F, Tez M. Red cell distribution width as a predictor of mortality in acute pancreatitis. Am J Emerg Med. 2013; 31(4):687-689.

[7] Oh J, Kang SM, Hong N, Choi JW, Lee SH, et al. Relation between red cell distribution width with echocardiographic parameters in patients with acute heart failure. J Card Fail. 2009; 15(6):517-522.

[8] Inuzuka R, Abe J. Red blood cell distribution width as a link between ineffective erythropoiesis and chronicinflammation in heart failure. Circ J. 2015; 79(5):974-985.

[9] Tseliou E, Terrovitis JV, Kaldara EE, Ntalianis AS, Repasos E, et al. Red blood cell distribution width is a significant prognostic marker in advanced heart failure, independent of hemoglobin levels. Hellenic J Cardiol. 2014; 55(6):457-461.

[10] Ani C, Ovbiagele B. Elevated red blood cell distribution width predicts mortality in persons with known stroke. J Neurol Sci. 2009; 277(1-2):103-108.
[11] Haltmayer M, Mueller T, Luft C, Poelz W, Haidinger D, et al. Erythrocyte mean corpuscular volume associated with severity of peripheral arterial disease: an angiographic evaluation. Ann Vasc Surg. 2002; 16(4):474-479.

[12] Demirtas S, Karahan O, Yazici S, Guclu O, Caliskan A, et al. The relationship between complete blood count parameters and Fontaine's Stages in patients with peripheral arterial disease. Vascular. 2014; 22(6):427-431.

[13] Bujak K, Wasilewski J, Osadnik T, Jonczyk S, Kołodziejska A, et al; The prognostic role of red blood cell distribution width in coronary artery disease. A review of the pathophysiology. Dis Markers. 2015; 2015:824624.

[14] Zalawadiya SK, Veeranna V, Niraj A, Afonso L, et al. Red cell distribution width and risk of coronary heart disease events. Am J Cardiol. 2010; 106(7):988-993.

[15] Isik T, Uyarel H, Tanboga IH, Kurt M, Ekinci M, et al. Relation of red cell distribution width with the presence, severity, and complexity of coronary artery disease. Coron Artery Dis. 2012; 23(1):51-56.

[16] Vaya A, Hernández JL, Zorio E, Bautista D. Association between red blood cell distribution width and the risk of future cardiovascular events. Clin Hemorheol Microcirc. 2012; 50(3):221-225.

[17] Zambon A, Pauletto P, Crepaldi G. Review article: The metabolic syndrome - a chronic cardiovascular inflammatory condition. Aliment Pharmacol Ther. 2005; (Suppl 2):20-23.

[18] Sánchez-Chaparro MA, Calvo-Bonacho E, González-Quintela A, Cabrera M, Sáinz JC, et al. Higher red blood cell distribution width is associated with the metabolic syndrome: results of the Ibermutuamur Cardiovascular Risk assessment study. Diabetes Care. 2010; 33(3):e40.

[19] Lippi G, Pavesi F, Bardi M, Pipitone S, et al. Lack of harmonization of red blood cell distribution width (RDW). Evaluation of four haematological analyzers. Clin Biochem. 2014; 47:1100-1103.

[20] Madjid M, Fatemi O. Components of the complete blood count as risk predictors for coronary heart disease. Tex Heart Inst J. 2013; 40(1):17-29.

[21] Wang H, Yang G, Zhao J. Association between mean corpuscular volume and severity of coronary artery disease in the Northern Chinese population: A cross-sectional study. J Int Med Res. 2020; 48(3):300060519896713. 